\title{
El «yo» en los Canti: preliminares al estudio de una instancia textual (II). El Discorso di un italiano intorno alla poesia romantica y el primer «yo» lírico leopardiano
}

\section{Francisco Amella Vela}

Universitat de Barcelona

\section{Resumen}

El artículo analiza algunas de las contradicciones internas del Discorso di un italiano intorno alla poesia romantica para explicar la escisión del primer yo lírico leopardiano en la paradójica dualidad del fanciullo / vecchio. La cuestión se aborda a la luz de las modificaciones que canzoni e idilli sufrieron a raíz de la constitución del libro de los Canti.

Palabras clave: Siglo XIX, crítica literaria, hermenéutica, poética, representación del «yo» lírico.

\section{Abstract}

This article analyzes some of the inner contradictions in Leopardi's Discorso di un italiano intorno alla poesia romantica as a way to explain the split of the first Leopardian lyrical subject into the paradoxical duality of a fanciullo / vecchio self. This question is viewed from the perspective of the rearrangement which canzoni and idilli went through when the Canti were constituted for the first time.

Key words: 19th Century, literary criticism, hermeneutics, poetics, representation of the lyrical subject.

La distinción entre canzoni e idilli resulta algo abusiva y engañosa cuando se aplica a los Canti, pues presenta como operativa en ellos una distinción entre géneros de la que su autor prefirió prescindir. ${ }^{1}$

1. Luigi Blasucci ha indicado que la elección del título Canti (en el que este término pierde su carácter técnico de composición verbal acompañada de música para indicar una "pura vocalità poetica») por parte de Leopardi constituye una verdadera innovación «all'interno delle istituzioni poetiche italiane, da parte di un autore che le conobbe come pochi altri», y persigue la unificación de los géneros líricos dentro del volumen. También Franco Gavazzeni se ha referido al hecho de que «la negazione di categorie formali tradizionalmente connesse a metri particolari» y el "raccogliere sotto il titolo complessivo di Canti specie metriche differenti» constituyen la "prima, drastica unificazione» que Leopardi lleva a cabo al publicar por primera vez sus Canti. Cfr. respectivamente, Luigi BlASUCCI, I titoli dei "Canti», 
Es dato que no se recuerda únicamente por escrúpulo de rigor o tempestividad terminológicos, sino por señalar la relevancia de ciertos rasgos estructurales del cancionero leopardiano: remitir, en referencia a él, a la neta distinción entre canciones e idilios equivale a mantener vigente el carácter de rígida alternativa que caracterizó los primeros proyectos editoriales de nuestro autor, pero que es ajena al espíritu de los Canti; por el contrario, la suspensión de las categorías genéricas restablece - para el lector, y para el poeta antes que para ningún otro lector - la compatibilidad de experiencias poéticas en apariencia inconciliables (y tradicionalmente así sentidas).

Es muy cierto que la opinión del propio autor acerca de la compatibilidad de ambas experiencias poéticas deja entrever, en ciertos momentos, fuertes reservas, cuando no un manifiesto escepticismo: es lo que se desprende del hecho de que las diera a conocer como líneas creativas independientes, antitéticas en su misma pariteticidad, ${ }^{2}$ en las series mutuamente excluyentes instituidas por las ediciones boloñesas de las Canzoni (1824) y de los Versi (1826). ${ }^{3}$

Y sin embargo, los Canti nos muestran ambas experiencias como conciliables. Por una parte, como hemos dicho, quedan abolidas las referencias a los géneros líricos (convertidas ahora todas las composiciones en "cantos»), y entrecruzados, en cierta medida, esos mismos textos que las ediciones boloñesas habían separado por géneros. Por otra, en el plano estilístico, la constitución del libro de los Canti supone la modificación, en grados diversos, de dichos textos, modificaciones tendentes a adecuarlos a los nuevos criterios formales que sucesivamente irán determinando la conformación del volumen, y a hacerlos, ante todo, más próximos entre sí de lo que, en realidad, ya eran.

En realidad, decimos: porque a pesar de las obvias diferencias que las distinguen, entre una y otra serie es posible percibir influencias recíprocas. Se ha

Napoli: Morano, 1989, p. 165-166; y Franco GAVAZZENI, «L'unità dei Canti: varianti e strutture», en "Feconde venner le carte». Studi in onore di Ottavio Besomi, Casagrande: Bellinzona, 1997, p. 447.

2. A la «pariteticità delle due esperienze» se refirió Domenico DE ROBERTIS en «Il sistema della poesia», en Giacomo LeOpardi, Canti, edizione a cura di Giuseppe e Domenico De RoberTIS, Milano: Mondadori, 1978, p. xxxi.

3. Respecto al volumen de las Canzoni, el de los Versi constituye un proyecto editorial enteramente distinto: a diferencia del principio cronológico que organiza el precedente proyecto editorial, el criterio antológico que organiza los Versi es precisamente la variedad de estilos y géneros. Con todo, el nuevo proyecto editorial se presenta como continuación de la obra presentada en la edición de 1824, tal como puede leerse en el prefacio de "gli editori a chi legge»: "Abbiamo creduto far cosa grata al Pubblico italiano, raccogliendo e pubblicando in carta e in forma uguali a quelle delle Canzoni del conte Giacomo Leopardi già stampate in questa città, tutte le altre poesie dello stesso autore, tra le quali alcune inedite, di cui siamo stati favoriti della sua cortesia.» Lo mismo se desprende de la explícita referencia del poeta a un proyecto, finalmente no realizado, de edición de las Opere del conte Giacomo Leopardi, del que informa a su hermano Carlo en carta fechada en Bolonia el 9 de noviembre de 1825 , en la que le pide que le remita, a efectos de dicha publicación, diversos manuscritos que se encontraban en Recanati. En ambos casos queda claro que Canzoni y Versi constituyen dos obras autónomas, que sólo pueden ser reunidas en un mismo volumen a condición de que éste sea de carácter compilatorio. 
señalado cómo la «experiencia idílica» contribuyó a disolver la rigidez estructural de las canciones, prestándoles una mayor concinnitas y afianzando, también en ellas, el empleo del endecasílabo libre (una de las constantes estilísticas de los idilios), ${ }^{4}$ que vendrá así a constituirse en marca poética referencial. ${ }^{5}$ Estos hechos bastan probablemente para explicar la solidaridad que, al constituir los Canti, el propio Leopardi percibió (o, siempre bien consciente de ella, decidió potenciar) entre unos textos y otros, portadores de afinidades más o menos recónditas.

Es cierto, en cualquier caso, que las afinidades que acabamos de señalar se hacen manifiestas tras una lectura, más que atenta, capilar, y que a primera vista no resulta en absoluto sencillo concebir en qué términos pudieron coexistir textos verdaderamente tan diversos, no ya en los Canti (donde las intervenciones del autor actúan, como se ha indicado, en el sentido de una cierta homogeneización formal), sino en el ánimo mismo del poeta, en la época de su composición. Pues, en efecto, no deja de resultar sorprendente que la «stagione idillica» conviva, o por lo menos coexista (pues a fin de cuentas se trata de coexistencia, por más que el problema pueda legítimamente plantearse en términos de alternativa) con la de las canzoni, coexistencia que se traduce en una especie de sorprendente desdoblamiento compositivo que nos muestra al poeta disponible a experiencias tan diferentes que más que presentarse como complementarias se dirían antagónicas.

No hay verdadera homología entre las divisiones internas que solemos establecer dentro de los Canti y las fases de la "carriera poetica» de Leopardi. Sin duda alguna, a los Canti se les debe reconocer un valor antológico, pues reunen prácticamente toda la producción, y lo más granado y característico, del Leopardi autor lírico. Mas la relación de especularidad vida-obra en la que se ha basado buena parte de las interpretaciones de los Canti no deja de resultar inadecuada si no se la matiza y maneja con cautela. ${ }^{6}$

4. «Endecasillabo sciolto, per chi abbia letto la poesia leopardiana anteriore al '22, significa automaticamente idilli. E in effetti i critici hanno messo in rilievo gli influssi che dal ciclo idillico (conclusosi, si ricordi, solo nell'estate-autunno del '21) si riverberano sulle canzoni». Cfr. Marco Santagata, Quella celeste naturalezza. Le canzoni e gli idilli di Leopardi, Bologna: Il Mulino, 1994, p. 68.

5. Cfr. Íbid., p. 67-68.

6. Aun a riesgo de parecer inmodesto, me permito remitir al lector de estas páginas a la primera parte de este trabajo, publicada en el número anterior de Quaderns d'Italià, donde me refiero, con una cierta amplitud, a esta cuestión. En definitiva, y lejos de rechazar la pertinencia de una consideración biográfica de la obra leopardiana (o de la de cualquier otro autor), el propósito que me mueve es el de señalar la conveniencia de no reducir la instancia textual del "yo» lírico a una identificación con la figura del poeta, ya se trate del poeta-hombre, ya del poeta-escritor (cual emerge de una lectura como la que realizara en su momento Giuseppe de Robertis), para avanzar en la interrogación del texto y todos sus sentidos plausibles a la luz del sujeto poético que genera el propio discurso lírico. 
Atendiendo a los datos biográficos, la crítica no ha podido dejar de constatar que en la historia de la escritura leopardiana hay un corte que permite establecer una primera escansión en la actividad literaria de nuestro autor: el corte introducido por el silencio en el que desemboca la poesía leopardiana en 1823, prolongado hasta $1828 .^{7}$ La fecha de 1823 se nos impone por su misma evidencia como la del extremo inferior que delimita esta primera «fase»o «etapa» de la poesía leopardiana, y la de 1828 como la del inicio de la fase sucesiva. Entre una y otra fecha, cinco años de un silencio que, en sí mismo, nada nos impide considerar como una verdadera fase de la poesía leopardiana; o como un elemento integrante y necesario de dicha primera etapa, por ser en realidad dicho silencio - el de la lírica - la modulación en timbres de prosa de la voz del poeta, la experiencia del primer gran desengaño que hará que esa voz madure y se haga más profunda.

Sin embargo, si no podemos dejar de señalar la existencia y el sentido de ese sintomático silencio, habremos de convenir también que en los Canti no se da silencio alguno, y que una cosa es interpretar en $\mathrm{Il}$ risorgimento las alusiones a cierto periodo de letargo (o de muerte simbólica), poético y existencial, como elipsis temporal en la «historia narrada» en los Canti (en cuyo caso, ¿quién es el sujeto de tal historia?), y otra distinta leerlas como referencia a los datos biográficos del autor (pero esa es, por cierto, otra historia).

Menos evidente es la fecha de inicio de la actividad poética del Leopardi «maduro»; y querer certificarla recurriendo a los Canti complica, más que resuelve, el problema. Pareciera que los Canti fijan un término a quo: el del año 1816, en que fue compuesta la "cántica" Appressamento della morte, la composición más temprana de las promovidas a cantos. Pero, en rigor, los versos de la cantica que se convierten en el fragmento XXXIX de los Canti constituyen, en sí mismos, un texto tardío. Il primo amore es también texto precoz (1817), pese a lo cual en los Canti aparece colocado no sólo después de las canciones patrióticas de 1818, sino detrás también de las escritas entre 1820 y 1822: no en vano Leopardi lo corrigió ampliamente de cara a su inclusión en la edición de los Versi, y aún habría de conocer nuevas enmiendas.

No parece, por lo tanto, que el ordenamiento de los Canti esté subordinado a la cronología de composición, por lo menos en lo que se refiere a las piezas ya existentes cuando los Canti ven la luz. Y si bien dicho ordenamiento no impediría postular la configuración, precisamente, de dos bloques sucesivos canzoni-idilli, la misma historia editorial de los Canti parece indicar en cualquier caso la mayor pertinencia de criterios distintos del de la adecuación a las fechas de composición o de la biografía del poeta para explicar la estructura conferi-

7. Como es sabido, después de componer el Inno ai Patriarchi, en julio de 1822, Leopardi dejará de escribir poesía durante más de un lustro, si exceptuamos Alla sua donna, de septiembre de 1823, los versos del coro de los muertos del Dialogo di Federico Ruysch e delle sue mummie, y la epístola Al conte Carlo Pepoli, escrita en 1826: en total, apenas (!) 245 versos en casi seis años. 
da al libro y las razones de inclusión, exclusión, reelaboración o recolocación en el mismo de las líricas que lo componen. ${ }^{8}$

Así pues, 1816 y 1823 serán, respectivamente, las fechas de inicio y clausura de la primera fase de la lírica leopardiana, mientras que en los Canti los «momentos» de inicio y final de una primera "fase» se encuentran, a su vez, en All'Italia y en Al conte Carlo Pepoli. Quedaría entonces por establecer, a propósito del problema de las fechas, de la historia de la lírica leopardiana y de la historia de los Canti, qué sentido haya que darle al término "fase», o «etapa», quizás no por fuerza esencialmente cronológico.

\section{3}

Así pues, ¿cuántas etapas se cierran en la carrera poética de Leopardi en 1823 ? $\mathrm{O}$, en otras palabras, los diversos géneros practicados por Leopardi a lo largo de esos siete años que van de 1816 a 1823 (la cantica, la canción, el idilio, la elegía), ¿constituyen una sola y la misma fase? O, si queremos, ¿una sola y la misma experiencia poética?

En realidad, y a pesar de las evidentes diferencias existentes entre una y otra, ambas experiencias líricas (canzoni y, más genéricamente, idilli) tratan de responder a las exigencias planteadas por Leopardi en su Discorso di un italiano intorno alla poesia romantica (de marzo de 1818) a quienes aspirasen a llamarse poetas, vale decir, en primer lugar, a sí mismo.

Las palabras conclusivas de dicho discurso «incitaban a la juventud italiana a devolver a la patria su primacía en el terreno de las artes y las letras y a resucitar en el degradado siglo presente el espíritu de la edad antigua».

Este «regeneracionismo programático» que impregna todo el Discorso no se limita, sin embargo, a la contemporánea situación italiana (dramática, en opinión de nuestro autor), aunque haya de aplicarse a ella con urgencia. De acuerdo con el Discorso, la degradación sobre la que Leopardi querría ver resurgir a la Italia de su tiempo es el resultado del inevitable (o sea histórico) empobrecimiento vivencial del hombre, empobrecimiento que nace de la mengua de su imaginación y su sensibilidad y del correlativo incremento de sus facultades intelectuales. En este sentido, uno de los muchos reproches que Leopardi dirige a los románticos en las páginas de su discurso es el de estar contribuyendo a reducir el horizonte de las experiencias del hombre moderno al querer limitar el espectro de la sensibilidad humana a la mera sentimentalidad o a

8. A propósito de la pertinencia del criterio cronológico como principio organizador en los Canti, cfr. Claudio Colaiacomo, "Canti, di Giacomo Leopardi», en Letteratura italiana. Le opere, vol. III. Dall'Ottocento al Novecento, Torino: Einaudi, 1995, p. 266: «Leopardi non si sentì particolarmente legato né all'ordine cronologico di composizione (e con esso all'idea di un parallelismo tra biografia reale e finzione poetica), né all'ordinamento (peraltro anche questo in larga misura rispettato) dei precedenti blocchi poetici (canzoni e idilli)».

9. Cfr. Giacomo LeOpardi, Cantos, edición bilingüe de María de las Nieves MuÑIz MuÑIz, Madrid: Cátedra, 1998, p. 99. 
la melancolía (lo melancólico patético), con la consiguiente reducción de todos los géneros poéticos al solo "patético»:

Ma quel ridurre pressoché tutta la poesia ch'è imitatrice della natura, al sentimentale, come se la natura non si potesse imitare altrimenti che in maniera patetica; come se tutte le cose rispetto agli animi nostri fossero sempre patetiche, come se il poeta non fosse più spinto a poetare da nessuna cosa eccetto la sensibilità, o per lo meno senza questa; come se non ci fosse più gioia non ira non passione quasi veruna, non leggiadria né dolcezza né forza né dignità né sublimità di pensieri; non ritrovato né operazione veruna immaginativa senza un colore di malinconico; questa cosa con che nome si dee chiamare? Dunque le cetre dei poeti avranno per l'avvenire una corda sola? E ciaschedun poema assolutamente e tutti rispettivamente saranno unisoni? dunque non ci saranno epopee, non canzoni trionfali, non inni non odi non canti di nessuna sorta se non patetici? [...] dunque Omero non fu poeta? o vero fu (come parve a molti che fosse), ma non è più? o vero è poeta e sarà, e diletta e diletterà supremamente, ma nessun poeta moderno dee cantare in quella forma? Ora come sarà disdetto ai poeti il cantare nella forma di Omero e di Pindaro $\mathrm{e}$ in breve degli antichi, finattantoché gli antichi diletteranno? [939a]. ${ }^{10}$

Se comprende, pues, que tanto el cultivo de un género fuertemente retórico como el de las canciones, cuanto la «disponibilidad» de Leopardi a practicar en un mismo periodo (1818-1823) géneros diversos (canzoni e idilli), más allá de la exhibición juvenil del talento y aún del virtuosismo, pretendan ser «en su momento, la fiel aplicación del programa enunciado pocos meses antes en el inédito Discorso di un italiano intorno alla poesia romantica». ${ }^{11}$

La lirica (poesia civile eloquente) è per l'appunto un esempio di quella poesia non-sentimentale di cui bisogna dotare l'Italia. Le canzoni patriottiche sono la risposta all'esigenza di una poesia moderna che recuperi i generi del passato espressa nel Discorso. ${ }^{12}$

Entre los autores italianos antiguos, Petrarca se alza en toda su estatura de maestro de nobles y no unísonas pasiones, y como modelo de una poligrafía sabiamente puesta al servicio de una sentimentalidad grande en todos los sentidos. El propósito que animará la producción lírica leopardiana en los años que siguen a la redacción del Discorso, y que orientará la «recuperación» de algunas de las composiciones compuestas antes de esa fecha, será por lo tanto el de demostrar

10. Se cita a partir de la edición Giacomo LeOPARDI, Tutte le opere, a cura di Walter BINNI y Enrico GHIDETTI, Firenze: Sansoni, 1985. La indicación corresponde al número de la página y a la relativa columna.

11. Cfr. María de las Nieves MuñIz MuñIz, Cantos, op. cit., p. 99.

12. Cfr. Marco SANTAGATA, Quella celeste naturalezza. Le canzoni e gli idilli di Leopardi, op. cit., p. 51. 
come la lettura moderna, anche romantica quindi, di un Petrarca «sentimentale» $[\ldots]$ potesse essere finalizzata alla proposta di un moderno petrarchismo, «sentimentale» non in senso psicologico, ma in senso retorico. Un petrarchismo, cioè, che lungi dall'essere imitazione $o$ anche solo ripresa di una manie$\mathrm{ra}$, testimoniasse la vitalità di una poesia civile che coniugava eloquenza $\mathrm{e}$ affettività, «stile»e «cuore». ${ }^{13}$

Si en el Discorso denunciaba la infelicidad del hombre por causa del progreso, Leopardi querrá ofrecer a los italianos el remedio de las pasiones fuertes, puras, intensas, como las que se viven por vez primera: las que experimentaron los primeros hombres, o las que experimenta todo hombre, antiguo o moderno, en la infancia y la primera juvenud. Esas pasiones debe imitar, y por ende suscitar en el lector, la poesía, a la cual se le asignaba en el Discorso la función de salvaguardar y transmitir "quanto di antico ancora sopravvive nel moderno e quanto di fanciullesco nell'adulto». ${ }^{14}$

Es en este punto donde cabe preguntarse en qué medida la primera poesía leopardiana, que es la historia de cómo "un poeta che "crede" fervidamente nella poesia possa ridursi al silenzio», ${ }^{15}$ consiguió realizar todas las aspiraciones que el joven poeta confiaba poder satisfacer. ¿Puede el lector experimentar en sus versos la plenitud de la comunión con la naturaleza? Leyendo las composiciones de esa primera «stagione» leopardiana se tiene por el contrario la impresión de que tal estado - ya sea recuerdo de la infancia individual, ya sea recuerdo del "poder» de la poesía entre los antiguos- pertenezca a un pretérito que más que remoto es un tiempo mítico o, peor aún, que debe ser identificado con un tiempo fabuloso en el que hipotéticas ruinas del futuro igualarían a las del pasado (con las que se abre la primera de las canzoni, y que volvemos a encontrar en $A d$ Angelo $M a i$ ), ruinas en las que la naturaleza recobra lo que la civilización le ha arrebatado, pero entre las cuales no hay rastro ni vislumbre de presencia humana:

Tempo forse verrà ch'alle ruine

Delle italiche moli

Insultino gli armenti, e che l'aratro

Sentano i sette colli e pochi Soli

Forse sien volti, e le città latine

Abiterà la cauta volpe, e l'atro

Bosco murmurerà fra le alte mura

(A un vincitore nel pallone, vv. 40-46). 
Alla Primavera constituye uno de los ejemplos más claros de esta imposibilidad por parte del hombre moderno de recuperar, ni siquiera por intercesión de la poesía, la dimensión de lo natural.

Es interesante observar cómo la primera intervención del «yo narrante», al que vemos en los últimos versos de la primera estrofa caracterizado como «anciano prematuro» («ed anco, Primavera odorata inspiri e tenti Questo gelido cor, questo ch'amara Nel fior degli anni suoi vecchiezza impara?»), da paso al comienzo de la segunda estrofa a la alusión a una situación que cabe suponer general (con sustitución de la primera por la tercera persona: «vivi e il disueto orecchio Della materna voce il suono accoglie?»), para transformarse en los versos finales de la canción en una primera persona plural que no deja dudas sobre su carácter de experiencia colectiva («Ma non cognato al nostro Il gener tuo [...] Tu le cure infelici e i fati indegni Tu dé mortali ascolta, Vaga natura, e la favilla antica Rendi allo spirto mio; e tu pur vivi, E se dé nostri affanni Cosa veruna in ciel, se nell'aprica Terra s'alberga o nell'equoreo seno Pietosa no, ma spettatrice almeno»), por más que el «yo» lírico no llegue a confundirse exactamente con ese «nosotros».

Presentar la situación del «yo» lírico como afín a la de sus lectores (o, en cualquier caso, a la de sus contemporáneos) es un modo de conjurar la proclividad de ese "yo" a presentarse como referente único del discurso poético: y es que la "affettività», el "cuore» exigen una constante vigilancia, para no arrastrar el dictado poético a la sima del patetismo romántico, límite éste siempre demasiado próximo y tentador para el hombre y el poeta moderno:

la soluzione "patetico-sentimentale» delle canzoni patriottiche portava a una impasse. Più in là del patetismo addolcito delle due [canzoni] rifiutate Leopardi non poteva spingersi. Se non altro, perché quel tipo di petrarchismo comportava che il discorso lirico si impostasse su un «io» onnipresente, che per sua natura tendeva a scivolare dalla funzione di elocutore a quella di protagonista e di soggetto di discorso. La storia successiva delle canzoni dimostra che molto presto Leopardi si svincola dall'ipoteca di quell'«io» che tutto filtra attraverso la soggettività autobiografica per tendere a un «noi», a un punto di vista collettivo, che consenta un'analisi «filosofica», cioè esterna e obiettiva, della realtà. Senza oggettivazione del discorso non ci sarebbero state neppure la canzoni civili. ${ }^{16}$

Si las canzoni, pues, reflejan la voluntad de Leopardi de proponer una reflexión de alcance universal, capaz de superar un subjetivismo reducido a individualidad, en las composiciones que integran el volumen de los Versi (idilios y elegías) la senda embocada difícilmente puede percibirse como distinta de la del autobiografismo, real o fingido. 
La pregunta se impone por sí sola: cuando canciones e idilios pasan a integrarse en los Canti, ¿puede decirse que una de esas dos instancias, la individual o la colectiva, prevalece sobre la otra? No aventuraremos una respuesta rotunda, que no sólo superaría las límitaciones de un discurso sobre los "preliminares al estudio de una figura textual», sino que no puede darse sin esclarecer antes cuál de ellas predomina en el conjunto de los cantos, y cómo debe interpretarse que lo haga. Y aún entonces habría que determinar si la instancia textual que gana en protagonismo cambia o permanece idéntica a sí misma de un extremo al otro del libro.

Sí podemos, en cambio, indicar por ahora lo siguiente: la incorporación, en 1835, de Il passero solitario, precisamente en esa zona inicial de los Canti en que a partir de 1831 conviven canzoni e idilli, parece indicar la acentuación del carácter «excepcional» (por su marginalidad) del «yo» lírico. Hay que determinar su peso específico: si contradice o desmiente, y en qué medida lo hace, la dimensión de lo colectivo, la eficacia del «nosotros» (y no sólo en el primer segmento de los Canti, sino en el conjunto de la obra).

Recordemos que lo que singulariza a este "yo» lírico es su naturaleza paradójica: protagonista y espectador a un tiempo, sabedor de su destino e incapaz de toda acción, joven, en fin, a la vez que viejo. Sorprende constatar que el germen de esta naturaleza contradictoria se encuentra, en realidad, en el Discorso di un italiano intorno alla poesia romantica. Mejor dicho: que el Discorso mismo nace de la paradoja que reúne en el hombre moderno al joven y al anciano, y ello pese al empeño puesto por Leopardi en negarlo.

Aunque tal vez no resulte inmediatamente evidente, la argumentación leopardiana del Discorso incurre en contradicción al sostener que el incremento de la razón es responsable del debilitamiento de la facultad imaginativa y un obstáculo para su restablecimiento, al tiempo que estima imprescindible el conocimiento de los clásicos (maestros, pues, no sólo de poesía, sino también de vida, en tanto que depositarios de las claves de la comunión con la naturaleza) para que el poeta moderno pueda dar cumplimiento a la tarea que se le encomienda en el Discorso. Como ha señalado Vincenzo De Caprio, ${ }^{17}$ esta contradicción deriva, en realidad, de la inicial inadvertencia leopardiana de la incompatibilidad entre las dos líneas que articulan sus reflexiones sobre la poesía de 1816 a 1818: la proclamación del carácter inmutable de la poesía y el reconocimiento de la especificidad de la poesía moderna. Esta inadvertencia se descubre manifiesta y potenciada en el carácter paradójico del «studio» que Leopardi encarece a quienes aspiran a llamarse poetas: en tanto que instrumento necesariamente arte factum para la recuperación de la espontaneidad del homo ignorans, la necesidad del estudio es el indicio más claro de la condición plenamente reflexiva del hombre moderno, pues supone la clara consciencia de la distancia que separa al moder-

17. Cfr. Vincenzo Di CAPRIO, «Senso della tradi,zione e poesia moderna in Giacomo Leopardi", en Letteratura e critica. Studi in onore di Natalino Sapegno, Roma: Bulzoni, 1974, p. 607-629. 
no, sabedor y — precisamente— «estudiado», del antiguo, «ignorante» y espontáneo.

Correlativo al de studio, el término senno hace, como aquél, su aparición en las reflexiones del Zibaldone que preceden a la redacción del Discorso; con él se quiere aludir a la conciencia que el poeta moderno tiene de su condición de poeta «no natural»:

Ma adesso l'arte è venuta in un incredibile accrescimento, tutto è arte e poi arte, non c'è più quasi niente di spontaneo, la stessa spontaneità si cerca a tutto potere ma con uno studio infinito senza il quale non si può avere [...] Questo avviene perché ora si viene da un tempo corrotto (oltreché si sta pure tra’ corrotti) e bisogna porre il più grande studio per evitare la corruzione, principalmente quella del tempo la quale prima che abbiamo pensato a guardarcene s'è impadronita di noi, e poi quella dei tempi passati, perché adesso conosciamo tutti i vizi delle arti e ce ne vogliamo guardare, e non siamo più semplici come erano i greci e i latini e i trecentisti e i cinquecentisti perché siamo passati pel tempo di corruzione e siamo divenuti astuti nell'arte [...] E però abbiamo molto più senno e arte che gli antichi, i quali per questo cadevano in infiniti difetti (non conoscendoli) in cui adesso non cadrebbe uno scolaro.[4] $]^{18}$

$\mathrm{Al}$ igual que sucede con studio (senno y studio vendrían a ser los dos momentos de una misma circunstancia: el primero sería, en particular, la lucidez respecto a la propia condición; el segundo, que nace de aquél, el remedio prescrito por la razón), el espesor de este concepto radica en su ambivalencia, ya que si ha sido precisamente el senno, es decir el incremento de discernimiento poético, lo que ha llevado a los poetas a la sofisticación y al artificio (como en el caso de esos románticos que quieren hacer de la poesía «tutt'uno colla metafisica» y convertir en materia de poesía el desvelamiento de los más recónditos lugares del alma), también es el senno lo que les permite advertir cuánto se han desviado del «commercio co" sensi» y corregir tal desviación en aras de una poesía que restablezca el vínculo del ser humano con la naturaleza.

Como ha indicado De Caprio, ${ }^{19}$ es este concepto de senno el que «comincia a far progredire il classicismo leopardiano verso una direzione nuova»; ante todo porque el carácter paradójico que acabamos de reconocer en él implica la reorganización conceptual del sistema de contrarios característico del pensamiento leopardiano: si la oposición, hasta este momento, había sido la usual en ámbito neoclasicista, o sea natura / arte como equivalente a naturalezza / artificio, a partir de ahora la oposición verdaderamente operativa va a ser inconsapevolezza / senno; de una argumentación en términos formales se pasa a un planteamiento que se resuelve en una dimensión no formalista.

Advirtamos, sin embargo, que si Leopardi encuentra la manera de abandonar la fe clasicista en el dogma de un aprendizaje técnico (entendido como

18. Se cita de la edición BINNI-GHIDETTI anteriormente referenciada. Como es costumbre, la indicación numérica corresponde a la de la página del original leopardiano.

19. Cfr. De CAPRIO, op. cit., p. 620 y 626. 
mera aprehensión y reproducción de la forma antigua) capaz de garantizar la equiparación con los modelos antiguos, logrando así imprimir un giro «antropológico" a sus reflexiones en torno a la poesía, ello se debe a que en las mismas páginas en las que aparecen los dos términos definitorios de la conciencia reflexiva del poeta moderno nuestro autor ha introducido también, «quasi marginalmente, non molto di più che un semplice paragone esplicativo», otro par opositivo que será «l'asse portante di tutta la speculazione leopardiana per un lungo periodo», el binomio fanciulli / vecchi, que se convertirá en la piedra de toque del Discorso y en el elemento antropológico que explica la originalidad de la postura leopardiana en el debate italiano entre románticos y neoclasicistas.

Es su carácter «natural» («natural» por contraposición al carácter cultural de los conceptos antiguo / moderno) lo que convierte a la oposición fanciullo I vecchio en la matriz conceptual en virtud de la cual es posible explicar en términos de "degeneración» la oposición entre la poesía antigua y la moderna y, más en general, entre la condición de sujeto antiguo y la de sujeto moderno (en definitiva, el carácter degenerativo de todo proceso natural, concepto al que Leopardi asimila el devenir de la historia); y a partir de la cual, por vía metafórica, recuperar y vivificar la trillada oposición entre la primavera y el invierno de la vida.

Cabría esperar, no obstante, que la aparición de este binomio, precisamente porque remite a una experiencia humana de validez universal («Ma che vo io cercando cose $[. .$.$] poco note, potendo dirne una [...] notissima a chicchessia,$ della quale ciascuno [...] mi debba essere testimonio? Imperocché quello che furono gli antichi siamo stati noi tutti [...] dico fanciulli e partecipi di quella ignoranza $[\ldots] »),{ }^{20}$ que introduce en la teorización leopardiana una coordenada previa por su naturaleza a toda especulación sobre las formas artísticas, tendría que abrir un resquicio a la posibilidad de una poesía moderna fruto de la recuperación directa de la «ignorancia infantil», esto es, una poesía exenta de la mediación que supone el estudio de los textos antiguos; Leopardi, por el contrario, insiste en el carácter inexcusable de este aprendizaje.

Entre la vivencia infantil de la condición natural y el proceso de desnaturalización al que la educación somete a todo individuo, existe, no obstante su antagonismo, un punto de intersección que explica por qué el poeta moderno necesita recuperar el imaginario de los antiguos. Este particular universo de representaciones, en cuanto acervo de lecturas tempranas, queda asociado a la experiencia misma del entusiasmo del fanciullo y, por lo tanto, se convierte en el instrumento más efectivo para que el adulto — el sujeto moderno- pueda volver a situarse en la perspectiva de «lo primitivo infantil»: en las imágenes de los antiguos, lo primitivo-infantil y lo primitivo-antiguo coinciden, y la «inclinación natural a lo primitivo» encuentra, por eso mismo, doble satisfacción; aunque dicha satisfacción constituya, en sí misma, una experiencia de cultura. 
Una experiencia, en consecuencia, paradójica, pues siendo como es demostración de una plenitud primigenia, sitúa no obstante dicha plenitud en un pasado doblemente mítico (porque se asocia a los mitos antiguos y porque pertenece a un tiempo irrecuperable): por ello Leopardi no puede dejar de repetir que la civilización moderna aleja necesariamente al ser humano de su naturaleza primera, privándole, en última instancia, de la capacidad para expresarla.

El «studio» es la prueba palmaria de que la forma antigua no es directamente transmisible ni sencillamente imitable: lo que posibilita el retorno a la condición de primitivismo es la experiencia de la poesía antigua a través de su estudio concienzudo y de su recreación, y tal experiencia adquiere su valor, en realidad, en cuanto reviviscencia de dicha condición de primitivismo.

Aquí es, entonces, donde el estudio descubre su naturaleza oximórica. Viene a ser como una esclusa cultural: como incremento de lo intelectivo incapacita para usar la palabra originaria; pero en cuanto vía de discernimiento, hace posible el reconocimiento de la palabra poética genuina y su re-creación.

La diferencia entre los antiguos poetas y los modernos existe, y no se reduce a la «desviación» romántica en el plano de lo poético: a fin de cuentas, también los anti-románticos son individuos modernos. Pero al llevar el debate entre unos y otros al terreno de la condición humana, Leopardi no hace sino ahondar la fractura que los separa. La insistencia del recanatense en la necesidad del estudio no es, por lo tanto, una mera rémora del formalismo clasicista, antes bien la consecuencia inevitable de la contradictoriedad misma de su fe en la posibilidad de un retorno ad origenes: una vez se ha situado la meta del retorno en una condición de primitivismo que convierte en equivalentes ignorancia infantil y espontaneidad primitiva (o viceversa, espontaneidad infantil e ignorancia primitiva), la superación de «la condizione artificiata dell'uomo moderno" sólo es posible afirmarla en el plano de la fantasía, y no en el de la razón, donde el engaño no es posible.

Es oportuno observar en este punto que los términos en que quedan definidas la condición del primitivo-fanciullo y del moderno-vecchio son los mismos en que apenas un año después de la redacción del Discorso será definida la noción de lo infinito. Recordemos, recurriendo a la formulación de Luigi Blasucci, que lo infinito para Leopardi es fruto o bien de la ignorancia o bien de la ficción imaginativo-fantástica. En una situación «natural», la ignorancia de los límites ciertos del mundo vendría a ser la condición necesaria, el correlato psicológico, de la infinitud supuesta del mundo; en la situación «artificiata» en la que se encuentra el individuo moderno a causa del conocimiento que le ha procurado la razón científica, el mundo ha dejado de ser infinito, y sólo a través de la ficción fantástica es posible concebirlo aún como indefinido (en la medida en que sus límites no siempre pueden ser fijados con precisión). Es decir, que sólo merced a un acto de autoengaño consentido puede el ser humano moderno volver a situarse en la perspectiva del homo ignorans, perspectiva que de otro modo le está vedada.

La tarea que Leopardi le encomienda a la poesía es, así pues, la «rottura fantastica della condizione artificiata dell'uomo moderno» mediante el «ritor- 
no fantastico» a la plenitud primordial; la poesía, sin embargo, como él mismo declara al inicio del Discorso, puede solamente inducir el engaño de la imaginación, no el del intelecto, que no puede borrar de sí lo que sabe, sino únicamente fingir transitoriamente que lo ha olvidado. En esto consiste la verdadera paradoja del hombre moderno (paradoja que hemos visto latente en la contradicción necesaria entre la inmutabilidad de la esencia humana y la especificidad del alma moderna, o entre la inmutabilidad del carácter de la poesía y la especificidad de la poesía moderna, o en la conciencia reflexiva inmanente a los términos senno y studio), que Leopardi descubre, se diría, a su pesar: que el regreso fantástico a la condición de plenitud de los antiguos (la condición de lo infinito) solo es posible en el plano de la ficción:

sapendo noi così tosto come, aperto un libro, lo vediamo scritto in versi, che quel libro è pieno di menzogne, e desiderando e proccurando quando leggiamo poesie, d'essere ingannati e nel metterci a leggere preparando e componendo quasi senza avvedercene la fantasia a ricevere e accogliere l'illusione, è ridicolo a dire che il poeta non la possa illudere quando non s'attenga alle opinioni e ai costumi nostri, quasi che noi non le dessimo licenza di lasciarsi ingannare più che tanto, e che ella non avesse forza di scordarsi né il poeta di farle scordare e opinioni e consuetudini e checchessia, non guardano che l'intelletto in mezzo al delirio dell'immaginativa conosce benissimo ch'ella vaneggia, e onnimamente e sempre tanto crede al meno falso quanto al più falso; ${ }^{21}$

lo cual, si bien se mira, explica el carácter inevitablemente sentimental —o sea moderno- de la poesía leopardiana.

\section{5}

La poética de la naturaleza (la poesía como fuente de ilusiones, estímulo a la actividad, revestimiento elocuente de un contenido generador de pasiones y de acciones magnánimas) y algunos otros de los postulados del Discorso perduran en efecto, como hemos visto, en la poética que dicta las canciones de Leopardi. Pero el Discorso acaba descubriendo su auténtica dimensión de toma de conciencia larvada, rechazada, negada incluso por parte de su autor, de la irreversibilidad del progreso y del afianzamiento de lo intelectivo, lo que en otras palabras significa que el advenimiento de la condición de modernidad es irreversible tanto para la humanidad en su conjunto como para cada individuo en particular.

También por las resonancias biográficas que encierra para nuestro poeta, ${ }^{22}$ este hecho constituye una especie de "trauma primordial» que no solo domi-

21. Íbid., p. 916b.

22. Recuérdense, a este propósito, las conocidísimas páginas 143-144 del Zibaldone, en las que nuestro autor detalla el proceso por el que «nella carriera poetica, il suo spirito ha percorso lo stesso stadio che lo spirito umano in generale». Dotado en un primer tiempo de un estado que era «allora in tutto e per tutto come quello degli antichi», el autor se ve transfor- 
na la reflexión teórica leopardiana acerca de la función de la poesía, sino que condiciona, de manera latente primero, consciente después, la constitución misma del primer "yo» lírico leopardiano (antes y después de la constitución de los Canti) y la posición que éste ocupa tanto frente al propio discurso lírico como, en particular, dentro de él.

Los Canti se revelan, como han demostrado algunos trabajos cuyas huellas sería interesante seguir, ${ }^{23}$ la constante reconsideración de esa psicología paradójica, siempre por delante de sí misma: no en vano el libro de los Canti nace de la doble refundación de la poesía y del «yo» lírico (como sin duda señala la articulación que, en el discurrir del libro de los Canti, establecen Al conte Carlo Pepoli e Il risorgimento) como queriendo señalar la identidad del canto con la voz que le da vida.

mado en «filosofo di professione (di poeta ch'io era)», es decir, ve consumado en sí mismo «il passaggio dallo stato antico al moderno».

23. Me refiero en particular a los interesantísimos trabajos, a mi juicio insuficientemente valorados, de Claudio Colaiacomo, ya citado; de Piero Bigongiari (Leopardi e il desiderio dell'io. Riflessioni preliminari sull'ordinamento dei "Canti», en «L'approdo letterario», 74, giugno 1976, p. 54-82); y de Vittorio PANICARA (La nuova poesia di Giacomo Leopardi. Una lettura critica della "Ginestra", Firenze: Olschki, 1997). A pesar de partir de premisas conceptuales y metodológicas bien diversas, en los tres trabajos el «yo» lírico ocupa una posición central, como verdadero eje vertebrador del cada uno de los cantos y del conjunto de los Canti, concebidos éstos como obra orgánicamente estructurada; los tres, además, postulan la existencia de un principio que podríamos denominar de "circularidad textual», responsable de sorprendentes efectos de sentido y que se explica, ante todo, por la centralidad del "yo» como figura ontológica y discursiva. 